\title{
White noise calculus and stocastic calculus
}

\author{
L. Accardi, Andreas Boukas*
}

AMS Mathematics Subject Classification 2000 : 60H40

\section{Introduction}

The plan of this paper is the following.

In section (2), after recalling some basic points of the structure theory of classical stochastic process, we illustrate how quantum probability can substantiate Hida's vision about the "elementality" of white noise, i.e. that any other (purely non deterministic) process can be in some sense "built" from it.

In section (3) we briefly mention how the idea of white noise approach to stochastic calculus was suggested by the stochastic limit of quantum theory and how it lead to the idea of developing a calculus for the non linear powers of white noise. In section (4 this idea is illustrated in the case of the square of white noise).

In section (5) we show how the classical processes, unified by the square of white noise emerged in different contexts of classical probability, mathematical physics, and statistics.

In section (6) we describe our attempt, developed in past years, to extend the results obtained for the square to higher powers of white noise. The

*Centro Vito Volterra, Università di Roma Tor Vergata, accardi@volterra.uniroma2.it WEB page: http://volterra.uniroma2.it Talk given at: International Conference on: Stochastic analysis: classical and quantum. Perspectives of white noise theory, Meijo University, Nagoya, November 1-5, 2003 Work done within the European Union Research Training Network (RTN): "Quantum Probability - Applications" web site: http://hyperwave.math-inf.uni-greifswald.de/algebra/qp_applications 
obstructions which make this dream hard to achieve (the so called no go theorems) are quickly reviewed in section (7).

Finally in the Appendix, following the original paper [Meix34], we give a proof of Meixner's classification theorem.

\section{$2 \quad$ Elemental processes}

The structure theory of classical stochastic processes is one of the highlights of classical stochastic analysis and it has been accomplished through the work of generations of probabilists. Cornerstones in this long and broad avenue have been the Wald decomposition; the De Finetti, Kolmogorov, Levy, Khintchin structure theory of independent increment processes; P. Levy martingale representation theorem and his innovation equation

$$
\delta X(t)=\Phi(X(s) ; s \leq t, Y(s), t, d t)
$$

which opened the way to Ito stochastic calculus and the corresponding theory of stochastic differential equations; the Hida-Cramer representation theory for Gaussian processes; the Kunita-Watanabe extension of Levy martingale representation theorem to non continuous trajectory martingales; the systematic work by Doob, Meyer and many others, ...

The intuitive picture emerging from these developments is that any "generic" stochastic process (i.e. whose trajectories are not too irregular) can be built starting from:

(i) deterministic processes $\left\{\dot{Z}_{t}\right\}$

(ii) distribution derivatives of stationary independent increment processes

in the sense that it can be decomposed as a sum of integrals of such processes.

Moreover, any stationary independent increment process $\left\{\dot{Z}_{t}\right\}$, in its turn, has the following Lévy-Ito decomposition :

$$
Z_{t}=m t+\sigma B_{t}+X_{t}
$$

where

$m$ is a constant

$B_{t}$ is a Brownian motion

$X_{t}$ is a compound Poisson process 
Finally any compound Poisson process $X_{t}$ can be expressed as an integral of independent Poisson processes $P_{u, t}$ with intensity of jumps equal to $u$

$$
X_{T}=\int_{0}^{T} d t \int P_{u, t} d \beta(u)
$$

and where the baricentric measure of this decomposition $d \beta(u)$ is called the Levy measure and has support in $\mathbb{R} \backslash\{0\}$.

These decompositions justify Hida's terminology according to which the i.e.r.v's (idealized elemental random variables ) are:

$\left\{\dot{B}_{t}\right\}$ the distribution derivatives of the standard Brownian motion

$\left\{\dot{P}_{u, t}\right\}$ the distribution derivatives of the standard Poisson process with intensity $u$

and this fact can be symbolically expressed by the formula:

$$
X_{t}=f\left(t,\{\dot{B}\},\left\{\dot{P}_{u}\right\}\right)=f\left(t,\left\{\dot{B}_{s}, s \in R\right\} ;\left\{\dot{P}_{u, s}, s \in \mathbb{R}, u \in \mathbb{R} \backslash\{0\}\right\}\right)
$$

expressing (in a unique way) the stochastic process $X_{t}$ as a (non-random) functional of:

(i) the standard deterministic processes: $t \mapsto t$

(ii) the standard white noise: $\dot{B}_{s}$

(iii) the standard Poisson processes with intensity $u \in \mathbb{R} \backslash\{0\}: \dot{P}_{u, s}$

Clearly equation (1) is purely symbolic because both $\dot{B}_{s}$ and $\dot{P}_{u, s}$ are random variable valued distributions and there is no natural way to define a nonlinear function of a distribution.

Notice moreover that, at this level of development, the standard white noise: $\dot{B}_{s}$ and the standard Poisson densities $\dot{P}_{u, s}$ appear on a totally equal level!

In the mid 1970's Hida initiated his programme on white noise analysis from which slowly it begun to emerge a more radical vision, namely:

there is only one elemental process: standard white noise!

I call this a "vision" because it was never formulated as a precise mathematical conjecture, nevertheless the outstanding role of white noise is a constant in Hida's mathematical thought [Hida01]. This is indeed a bold vision, because apparently the noises $\dot{B}_{s}$ and $\dot{P}_{u, s}$ look like totally disjoint objects!

The first substantial support to Hida's vision came from Hudson and Parthasarathy discovery [HuPa84b] that, from the quantum probabilistic 
point of view, the standard Poisson process with intensity $u$ can be considered as a "function" of the quantum white noise. More precisely (cf. the end of this section for the definitions involved):

Theorem: Let $P_{u, t}$ denote the classical scalar valued standard Poisson process with intensity $u$. Then

$$
\dot{P}_{(u, t)}=\sqrt{u} \dot{B}_{t}+\sqrt{u} \dot{B}_{t}^{+}+\dot{B}_{t}^{+} \dot{B}_{t}
$$

This achievement was an important event for probability and created a great impression in classical probabilists such as P.A. Meyer, because it showed that the Brownian motion and the Poisson process which, in classical probability, are not apparently connected, in quantum probability become expressible as sums of three fundamental objects (two if one considers, as usual in physics, the pair $\left\{\dot{B}_{t}, \dot{B}_{t}^{+}\right\}$as a single object: the quantum Brownian motion)

Notice however that the number process and the quantum Brownian motion are independent in the very strong sense that a sum of stochastic integrals over these processes is zero if and only if each integrand is zero. In other terms: the white noise point of view is necessary in order to achieve the full reductionistic programme (Hida's vision) of expressing the two building blocks of classical stochastic processes (Wiener and Poisson) as functions of a single more fundamental object: the quantum white noise. Notice in addition that, in order to achieve this reduction one needs the "square modulus of the white noise" $\dot{B}_{t}^{+} \dot{B}_{t}=\left|\dot{B}_{t}\right|^{2}$ (which is for the number process what the white noise is for Brownian motion). This "quadratic functional of the white noise" is the first example of the basic role played by the "nonlinear powers the white noise".

Using the above reduction Parthasarathy [Parth92] proved that any infinitely divisible distribution can be represented as vacuum distribution of a stochastic process in the space of the Boson Fock Brownian motion.

This result has now been developed into one of the basic new ideas of quantum probability: the quantum decomposition of a classical random variable [AcBo97].

Another important step in the direction of Hida's vision will be discussed in the following section. 


\section{White noise approach to classical and quan- tum stochastic calculus}

The white noise approach to classical and quantum stochastic calculus came out from the experience accumulated in the solution of many concrete problems in physics and in mathematics. The main steps in this development are:

1) The development of quantum stochastic calculus: Hudson and Parthasarathy (1982)

2) The proof that quantum stochastic differential equations are "fast time" $\left(t \rightarrow t / \lambda^{2}\right)$ limits of Hamiltonian equations: Accardi, Frigerio, Lu (1987)

3) The proof that white noise Hamiltonian equations are "fast time" limits of Hamiltonian equations: Accardi, Lu, Volovich (1993)

4) The combination of 2) and 3) into the statement that (classical and quantum) stochastic equations can be expressed as (causally) normally ordered forms of (classical and quantum) white noise Hamiltonian equations [AcLuVo99], [AcLuVo02].

The equivalence between white noise Hamiltonian equations and stochastic differential equations is quite nontrivial and the connection between the coefficients of the two types of equations is strongly nonlinear.

The advantage of the white noise Hamiltonian equations over their (classical or quantum) stochastic equivalent is that the formal unitarity conditions are the obvious ones, used by every physicist, which correspond to the formal self-adjointness of the Hamiltonian. The relationship expressing the coefficients of the stochastic equation as (nonlinear) functions of the coefficients of the corresponding white noise Hamiltonian equation explains the deep origins of the Hudson-Parthasarathy unitarity conditions and gives a "microscopic" interpretation of them in terms of the original (non stochastic neither white noise) Hamiltonian equations of which the stochastic and white noise equations are approximations.

A systematic development with several new results can be found in the PhD thesis of Wided Ayed [Ayed05].

Recall that the standard scalar valued, classical white noise is a classical mean zero, Gaussian, operator valued distribution process with variance

$$
\left\langle w_{s} w_{t}\right\rangle=\delta(t-s)
$$


This implies that the standard white noise is a stationary, additive, independent increment process.

Definition: A quantum stochastic process is a family

$$
x=\left\{x(t): t \in \mathbf{R}^{d}\right\}
$$

of Hilbert space operators. Such a process is said to be classical if

(i) For all $t \geq 0$, each $x(t)$ is a QM "observable" i.e

$$
x(t)=x(t)^{*} \quad ; \quad \forall t
$$

(ii) For all $t, s$,

$$
[x(t), x(s)]=x(t) x(s)-x(s) x(t)=0
$$

the commutator (here and in the following) will always be meant weakly on some dense domain.

Definition: A boson Fock ( $d$-dimensional) white noise (equivalently called, in the physical literature, a free boson Fock field over $L^{2}\left(\mathbb{R}^{d}\right)$ ) is a pair $b_{t}, b_{s}^{+}$of operator valued distributions acting on a Hilbert space $\mathcal{H}$, satisfying the commutation relations:

$$
\left[b_{t}, b_{s}^{+}\right]=\delta(t-s) \quad t \in \mathbb{R}^{d} \quad \text { [algebra] }
$$

and such that there exists a unit vector $\Phi \in \mathcal{H}$ satisfying:

$$
b_{t} \Phi=0 \quad \text { [Fock prescription] }
$$

For the precise meaning of these expressions, including domains, etc. ... cf. [AcAyOu03].

The definition above best illustrates one of the basic general principles of QP namely that:

algebra implies statistics.

In fact the above definition of white noise, which is purely algebraic, implies the Brownian motion statistics in the sense that it implies that the random variables

$$
W_{t}-W_{s}:=\int_{s}^{t} d u\left(b_{u}^{+}+b_{u}\right) \quad ; \quad W_{0}=0
$$

mutually commute, are independent on disjoint intervals and

$$
\left\langle\Phi, e^{i W_{t}} \Phi\right\rangle=\left\langle\Phi, e^{i \int_{0}^{t} d s\left(b_{s}^{+}+b_{s}\right)} \Phi\right\rangle=e^{-\frac{t^{2}}{2}}
$$


equivalently:

$$
W_{t}=B_{t}+B_{t}^{+}=\int_{0}^{t} d s\left(b_{s}^{+}+b_{s}\right)
$$

is the increment process of a classical Brownian motion (this is the quantum decomposition of the classical Brownian motion). Similarly one has the quantum decomposition of the classical white noise

$$
w_{t}=b_{t}+b_{t}^{+}
$$

In this sense one can say that in classical probability $w_{t}$ is "elemental" (atomic), but in quantum probability it is not.

The connection between (the increment process of) quantum Brownian motion and quantum white noise is the same as in the classical case:

$$
B_{t}^{+}=\int_{0}^{t} d s b_{s}^{+} \quad, \quad B_{t}=\int_{0}^{t} d s b_{s}
$$

Conversely a naive approach would suggest the conjecture that the relation between quantum Brownian motion and quantum white noise is:

$$
b_{t}:=\frac{d}{d t} B_{t} \quad, \quad b_{t}^{+}:=\frac{d}{d t} B_{t}^{+}
$$

However application of this rule without constraints leads to the wrong results as illustrated by the Hamiltonian white noise equations.

\section{Nonlinear powers of white noise}

Given the identification between the standard ( $d$-dimensional) quantum white noise and the free boson Fock fields over $L^{2}\left(\mathbb{R}^{d}\right)$, one can formulate what has been one of the fundamental unsolved problems of theoretical physics since over 30 years:

to give a reasonable construction of "local powers of free quantum fields".

This means that one would like to associate well defined mathematical objects to symbolic expressions of the form:

$$
w_{t}^{n}, b_{t}^{h}, b_{t}^{+k}
$$


in such a way to preserve as many properties as possible of the discrete approximations of these objects (which are well defined!). In other terms we would like to give a meaning to the ill defined "powers of an operator valued distributions". Since these objects are very singular some "renormalization" is needed to achieve this goal.

In the literature one can find many "renormalization techniques". The usual technique consists in introducing appropriate cut-offs and then trying to remove them with some limiting procedure and after having subtract some quantities tending to $\infty$. The main problem met up to now with this technique is the following dichotomy. After renormalization the resulting field is:

(i) either trivial (Gaussian or simple perturbation thereof)

(ii) or completely uncontrollable

This dichotomy is well illustrated in the paper by Segal [Sega70a] which is devoted to the simplest renormalization problem: that involving the squares of quantum fields.

Segal proves that the square of the usual time zero, scalar, Fock, KleinGordon field on $\mathbb{R}^{d}$, cannot correspond to a self-adjoint operator acting on the same Fock space of the field unless $d=1$. However his techniques are not able to provide:

- any information where this operator lives

- any information on its spectral distribution in interesting states

Thus, even for squares of fields the situation was very obscure until 1999. In 1999 a new idea was introduced in this problem. The main point of this new idea was to postulate new, renormalized, commutation relations for the higher powers of white noise and then construct some Hilbert space realization of them. More precisely: one starts from the standard boson algebra

$$
\left[b_{t}, b_{s}^{+}\right]=\delta(t-s)
$$

and computes formally the higher powers commutators

$$
\left[b_{t}^{h}, b_{s}^{+k}\right]
$$

combining the 1-st order commutation relations with the renormalization rule:

$$
\delta(t-s)^{n}=c^{n-1} \delta(t-s) \quad \forall n \in \mathbb{N} \backslash\{0\}
$$

Then one takes the result of this manipulation as the definition of the Lie algebra of the renormalized higher powers of white noise. 
The second much more difficult step consists in trying to construct some Hilbert space realization, for example by introducing the Fock prescription:

$$
b_{t}^{+k} b_{t}^{h} \Phi=0 \quad ; \quad h \geq 1
$$

which, also in this case, uniquely determines the statistics.

For the second powers, in the Fock case, this programme was realized in [AcLuVo99] and led to the definition of the renormalized square of white noise (RSWN) as the Lie algebra with generators

$$
\begin{gathered}
b_{\varphi}^{+} "=" \int d t \varphi(t) b_{t}^{2} ; \quad b_{\varphi}=\left(b_{\varphi}^{+}\right)^{+} \\
n_{\varphi} "=" \int d t \varphi(t) b_{t}^{+} b_{t}
\end{gathered}
$$

where $\varphi$ is some test function, and Lie brackets given by the commutation relations:

$$
\begin{gathered}
{\left[b_{\varphi}, b_{\psi}^{+}\right]=\gamma\langle\varphi, \psi\rangle+n_{\bar{\varphi} \psi}} \\
{\left[n_{\varphi}, b_{\psi}\right]=-2 b_{\bar{\varphi} \psi}} \\
{\left[n_{\varphi}, b_{\psi}^{+}\right]=2 b_{\varphi \psi}^{+}} \\
\left(b_{\varphi}^{+}\right)^{+}=b_{\varphi} \quad ; \quad n_{\varphi}^{+}=n_{\bar{\varphi}}
\end{gathered}
$$

where $\gamma$ is a strictly positive constant called "the renormalization constant".

The Fock representation of this Lie algebra consists in realizing these operators on a Hilbert space $\mathcal{H}$ with a unit vector $\Phi$ satisfying:

$$
b_{\varphi} \Phi=0
$$

Theorem: (Accardi, Lu, Volovich 1999) The Fock representation of the renormalized square of white noise (RSWN) exists.

Soon after this result it was recognized by Accardi, Franz and Skeide [AcFrSk00] that the Lie algebra of the renormalized square of white noise is isomorphic to (a central extension of) $\operatorname{sl}(2, \mathbb{R})$. This has 3 generators

$$
B^{-}, \quad B^{+}, \quad M
$$

and relations

$$
\left[B^{-}, B^{+}\right]=M
$$




$$
\left[M, B^{ \pm}\right]= \pm 2 B^{ \pm}
$$

The paper [AcFrSk00], combining the well developed theory of unitary representations of $s l(2, \mathbb{R})$ with Schürmann's representation theorem for quantum independent increment processes [Schü93], obtains:

(i) a classification of all the unitary representations of this current algebra satisfying an irreducibility condition

(ii) a classification of all the classical sub-processes of the RSWN as well as the identification of their vacuum distributions.

An unexpected fall out of this identification was an unexpected connection with the Meixner classes [Meix34].

In order to illustrate this connection let us first consider the case of the 1st order quantum white noise $b_{t}^{+}, b_{t}$ and the family of classical (self-adjoint) processes that can be obtained by linear combinations from it and the numbers process $b_{t}^{+} b_{t}$. A simple calculation shows that these processes are of the form

$$
x(t)=\alpha+z B(t)+\bar{z} B^{+}(t)+\beta N(t)
$$

where $\alpha, \beta \in \mathbb{R}$ and $z \in \mathbb{C}$. In more usual white noise notations:

$$
\dot{x}(t)=\alpha+z b_{t}+\bar{z} b_{t}^{+}+\beta b_{t}^{+} b_{t}
$$

The constant part $\alpha$ is central and we omit it. Also the case $z=0$ is trivial and we omit it. Then, up to the "time rescaling" $t \mapsto t /|z|$, the "gauge transformation" $b_{t} \mapsto e^{i \theta} b_{t}$, where $z=|z| e^{i \theta}$ and a renaming of $\beta$, we are left with the family

$$
\dot{x}(t)=b_{t}+b_{t}^{+}+\beta N(t)
$$

Remembering the Hudson-Parthasarathy decomposition of the white and Poisson noises, we see that, depending on the parameter $\beta$, this family only 1 critical case, namely: $\beta=0$ which corresponds to classical scalar valued standard Brownian motion

$$
x(t)=b(t)+b^{+}(t)
$$

If $\beta \neq 0$ then, fixing a complex square root of

$$
\sqrt{\lambda}=\sqrt{\beta^{-1}}
$$

after another rescaling and gauge transformation we obtain:

$$
x(t)=\sqrt{\lambda} B(t)+\sqrt{\lambda} B^{+}(t)+N(t)
$$


which is the classical scalar valued standard Poisson process with intensity $\lambda$.

A similar calculation applied to the RSWN shows that the family of classical processes that can be obtained by linear combinations from the $2-\mathrm{d}$ order processes $b_{t}^{+2}, b_{t}^{2}$ and the numbers process $b_{t}^{+} b_{t}$ has the form:

$$
x(t)=\alpha+z b_{t}^{2}+\bar{z} b_{t}^{+2}+\beta b_{t}^{+} b_{t}
$$

where $\alpha, \beta \in \mathbb{R}$ and $z \in \mathbb{C}$. Again up to centering, rescaling and a gauge transformation:

$$
x_{\beta}(t):=b_{t}^{+2}+b_{t}^{2}+\beta b_{t}^{+} b_{t}=B_{t}^{+}+B_{t}+\beta N_{t}
$$

where $\beta$ is a real number. It can be proved that here there are 2 critical cases:

$$
\beta= \pm 2
$$

the value +2 corresponding to the square position (classical) white noise, i.e. $\left|b_{t}^{+}+b_{t}\right|^{2}=b_{t}^{+2}+b_{t}^{2}+b_{t}^{+} b_{t}+b_{t} b_{t}^{+}+=b_{t}^{+2}+b_{t}^{2}+2 b_{t}^{+} b_{t}+\delta(0) \equiv b_{t}^{+2}+b_{t}^{2}+2 b_{t}^{+} b_{t}$ and the value -2 to the renormalized square of the momentum white noise, i.e.

$$
\left(b_{t}^{+}-b_{t}\right) / i
$$

the vacuum distribution of both these critical processes is the Gammadistribution

$$
\mu(d x)=\frac{|x|^{m_{0}-1}}{\Gamma\left(m_{0}\right)} e^{-\beta x} \chi_{\beta \mathbb{R}_{+}}
$$

whose parameter $m_{0}>0$ is uniquely determined by the choice of the unitary representation of $\operatorname{sl}(2, \mathbb{R})$ corresponding to the representation of the renormalized square of white noise algebra [ACFRSK00]

In this functional realization the number vectors become the Laguerre polynomials which are orthogonal for the gamma distribution.

Since the Gamma-distributions are precisely the distributions of the $\chi^{2}$ random variables, this result confirms the naive intuition that the distribution of the renormalized square of white noise should be a Gamma-distributions

For $|\beta|<2$ the jumps are not strong enough and one still has a density

$$
\mu(d x)=C \exp \left(-\frac{(2 \arccos \beta+\pi) x}{2 \sqrt{1-\beta^{2}}}\right)\left|\Gamma\left(\frac{m_{0}}{2}+\frac{i x}{2 \sqrt{1-\beta^{2}}}\right)\right|^{2}
$$


where $C$ is a normalization constant. The orthogonal polynomials corresponding to this probability measure are the of the second kind, or MeixnerPollaczek polynomials.

For $m_{0}$ integer or half-odd there are explicit formulae for the densities due to Grigelionis:

$$
\begin{gathered}
|\Gamma(n+i x)|^{2}=\frac{\pi x\left(1+x^{2}\right) \ldots\left((n-1)^{2}+x^{2}\right)}{\sinh (\pi x)}, n=1,2, \ldots,, x \in \mathbb{R} \\
\left|\Gamma\left(\frac{1}{2}+i x\right)\right|^{2}=\frac{\pi}{\cosh (\pi x)}, x \in \mathbb{R} \\
\left|\Gamma\left(n+\frac{1}{2}+i x\right)\right|^{2}=\frac{\pi\left(\frac{1}{4}+x^{2}\right) \ldots\left((n-1) n+\frac{1}{4}+x^{2}\right)}{\cosh (\pi x)} \quad, \quad n=1,2, \ldots ; x \in \mathbb{R}
\end{gathered}
$$

Finally, for $|\beta|>2$ the jumps dominate and the probability measure is atomic, namely the negative binomial (Pascal) distribution:

$$
\mu=C \sum_{n=0}^{\infty} \frac{c^{2 n}\left(m_{0}\right)_{n}}{n !} \delta_{\operatorname{sgn}\left(\beta\left((c-1 / c)\left(n+m_{0} / 2\right)\right)\right.}
$$

where $\left(m_{0}\right)_{n}$ denotes the Pochammer symbol

$$
\left(m_{0}\right)_{n}=m_{0}\left(m_{0}+1\right) \cdots\left(m_{0}+n-1\right)
$$

and

$$
\begin{gathered}
C^{-1}=\sum_{n=0}^{\infty} \frac{c^{2 n}\left(m_{0}\right)_{n}}{n !}=\left(1-c^{2}\right)^{-m_{0}} \\
c=\beta / 2-\sqrt{(\beta / 2)^{2}-1}
\end{gathered}
$$

if $\beta>+2$

$$
-\beta / 2-\sqrt{(\beta / 2)^{2}-1}
$$

if $\beta<-2$.

The orthogonal polynomials associated to a centered Pascal (negative binomial) distribution are the Meixner polynomials of the first kind:

$$
P_{n}(x)=(-1)^{n} \prod_{k=1}^{n} \frac{n+m_{0}-1}{n} M_{n}\left(\frac{x}{c-1 / c}-\frac{m_{0}}{2} ; m_{0} ; c^{2}\right)
$$


if $\beta>+2$ and

$$
\prod_{k=1}^{n} \frac{n+m_{0}-1}{n} M_{n}\left(-\frac{x}{c-1 / c}+\frac{m_{0}}{2} ; m_{0} ; c^{2}\right)
$$

if $\beta<-2$.

\section{Emergence of the square of white noise in different contexts}

The connection between the gamma processes and the current representations of $S L(2, \mathbb{R})$ was studied in [TsiVeYo01] independently of [AcFrSk00] (where this connection was established for all the Meixner classes).

The Lévy processes, corresponding to the Pascal measures were introduced by Bruss and Rogers [BruRo91] in the context of optimal selection strategies based on relative ranks, when the total number of options is unknown.

In the paper [Grig01] Grigelionis uses the term "Meixner distribution" to denote the class of probability measures on $\mathbb{R}$ whose characteristic function (Fourier transform of the probability density) has the form

$$
\hat{f}(z)=\left(\frac{\cos (\beta / 2)}{\cosh ((\alpha z-i \beta) / 2)}\right)^{2 \delta}
$$

with $z \in \mathbb{R},-\pi<\beta<\pi, \delta>0, \mu \in \mathbb{R}$. This class of probability measures was introduced by Schoutens and Teugels [SchTeu98] who established their connection with the Meixner-Pollaczek polynomials and proved that the measures in this class correspond to Levy processes (the explicit construction of the Fock representation in [AcLuVo99] can be considered a different proof of this result).

The papers by Nualart and Schoutens [NUSCH00] and by Schoutens and Teugels [SchTeu98] study the gamma, Pascal, and Meixner processes as main examples of generalized chaotic representation for square-integrable random variables in terms of the orthogonalized Teugels martingales (which are the centered power jump processes related to the original process). They use the one-dimensional polynomials of Meixner's type in order to carry out the orthogonalization procedure of the Teugels martingales . 
We refer to [Grig01], [Grig99], [Grig00c] for several interesting properties of these distributions and explicit formulae related to them.

In particular, in [Grig01], the Meixner process was proposed as a model for risky assets and an analogue of the Black and Sholes formula was established for them.

The infinite dimensional and multidimensional analogues of orthogonal polynomials associated to a given measure have been widely studied both in the Gaussian case and in the Poisson case (CHIHA, KOKUOL).

The programme to extend this analysis to more general probability measures was developed by Berezansky [BEREZb98], [BEREZa97], [BEKO94], [BELILY95] who introduced in this connection the notion of Jacobi field of operators, and his school [LYTV02a], [LYTV02b], [LYTV95c].

An infinite-dimensional analogue of the Laguerre polynomials and the associated Jacobi fields, corresponding to the gamma case, i.e. to the class (III) in Meixner's classification, was studied in [KonLit00], [KoSiStr97].

In conclusion it should be added that the square of white noise (RSWN) was introduced as an example of interacting Fock space and in the attempt to extend to infinite dimensions the canonical connection between orthogonal polynomials and interacting Fock spaces established, in the 1-dimensional case, in [AcBo97] and, in the 1-dimensional case, in [AcKuSt02].

\section{$6 \quad$ Higher powers of white noise}

The next step of our programme is: to extend, if possible, the results obtained for the square to higher powers of white noise. We are developing this programme jointly with Andreas Boukas including collaborations with Uwe Franz, in the attempt to overcome the obstructions posed by the no-go theorems (cf. below), and with Rene Schott and Massimo Regoli, on Lie algebra and algorithmic aspects: among other things we are trying to extend the symbolic programme developed by Feinsilver and Schott [FeinScho93] for calculations on Lie algebras.

Our programme is to characterize those independent increment stationary processes (SIIP or Levy processes) which arise as renormalized higher powers of the standard (Fock) quantum white noise. For a short period, in 2003, we believed we had realized this dream but, as I will try to explain below, the situation is more subtle and the problem is related to some long standing open problems in the classical theory of SIIP. 


\section{Definition}

The renormalized boson Fock white noise ( simply RBF white noise in the following ) over a Hilbert space $\mathcal{H}$ with vacuum (unit) vector $\Phi$ is the locally finite Lie $*$-algebra canonically associated to the associative unital $*$-algebra of operator-valued distributions on $\mathcal{H}$ with generators

$$
b_{t}^{\dagger n}, \quad b_{t}^{k}, \quad k, n \in \mathbf{N} \quad, \quad t \in \mathbf{R}^{d}
$$

and relations

$$
\begin{gathered}
{\left[b_{t}, b_{s}^{\dagger}\right]=\delta(t-s)} \\
{\left[b_{t}^{\dagger}, b_{s}^{\dagger}\right]=\left[b_{t}, b_{s}\right]=0} \\
\left(b_{s}\right)^{*}=b_{s}^{\dagger} \\
b_{t} \Phi=0 \\
\delta(t)^{l}=c^{l-1} \delta(t) \quad, c>0 \quad, l=2,3, \ldots
\end{gathered}
$$

Lemma The Lie algebra, associated to the RBF white noise (renormalized boson Fock white noise), is the Lie algebra with generators

$$
b_{t}^{\dagger k} b_{t}^{n}=: b_{n}^{k}(t)
$$

central element $b_{t}^{0} b_{t}^{\dagger 0}=: E$ and relations

$$
\begin{gathered}
\left(b_{s}^{\dagger k} b_{t}^{n}\right)^{\dagger}=\left(b_{t}^{\dagger n}\right) b_{s}^{k} \\
{\left[b_{t}^{n}, b_{s}^{\dagger}\right]=\epsilon_{n, 0} \epsilon_{k, 0} \sum_{l \geq 1}\left(\begin{array}{c}
n \\
l
\end{array}\right) k^{(l)} c^{l-1} b_{s}^{\dagger}{ }^{k-l} b_{t}^{n-l} \delta(t-s)}
\end{gathered}
$$

where: $k=0,1,2, \ldots$,

$$
\begin{gathered}
\epsilon_{n, k}:=1-\delta_{n, k} \\
k^{(l)}=k(k-1)(k-2) \cdots(k-l+1)
\end{gathered}
$$

$k^{(l)}=0$ if $l>k$

$\left(\begin{array}{l}n \\ l\end{array}\right)=0$ if $l>k$

These conditions guarantee that no negative powers of the white noise functionals appear.

In terms of the smeared generators

$$
B_{k}^{n}(f)=\int_{\mathbb{R}^{d}} f(t) b_{t}^{\dagger^{n}} b_{t}^{k} d t
$$


with involution

$$
\left(B_{k}^{n}(f)\right)^{*}=B_{n}^{k}(\bar{f})
$$

and central elements

$$
B_{0}^{0}(f)=E \int_{\mathbf{R}^{d}} f d t
$$

the relations become

$$
\begin{gathered}
{\left[B_{K}^{N}(\bar{g}), B_{k}^{n}(f)\right]=\epsilon_{K, 0} \epsilon_{n, 0} \sum_{L \geq 1}\left\{\left(\begin{array}{c}
K \\
L
\end{array}\right)\right\} n^{(L)} c^{L-1} B_{K+k-L}^{N+n-L}(\bar{g} f)-} \\
-\epsilon_{k, 0} \epsilon_{N, 0} \sum_{l \geq 1}\left\{\left(\begin{array}{l}
k \\
l
\end{array}\right)\right\} N^{(l)} c^{l-1} B_{K+k-l}^{N+n-l}(\bar{g} f)
\end{gathered}
$$

Now let us deduce some necessary conditions for the existence of the Fock representation.

Lemma (Boson Independent increments) Suppose that in the scalar product

$$
\left\langle B_{0}^{K_{N}}\left(f_{N}\right) \ldots B_{0}^{K_{1}}\left(f_{1}\right) \Phi, B_{0}^{n_{M}}\left(g_{M}\right) \ldots B_{0}^{n_{1}}\left(g_{1}\right) \Phi\right\rangle
$$

the supports of any two test functions either coincide or are disjoint.

Denote by $\mathcal{I}$ the family of all supports of all the test functions appearing in the scalar product. Then the above scalar product is equal to

$$
\prod_{I \in \mathcal{I}}\left\langle\prod_{\left\{h: \operatorname{supp}\left(f_{h}\right)=I\right\}} B_{0}^{K_{h}}\left(f_{h}\right) \Phi, \prod_{\left\{k: \operatorname{supp}\left(g_{k}\right)=I\right\}} B_{0}^{K_{k}}\left(g_{k}\right) \Phi\right\rangle
$$

where if $\left\{\lambda: \operatorname{supp}\left(\phi_{\lambda}\right)=I\right\}=\emptyset$ we interpret

$$
\prod_{\left\{\lambda: \operatorname{supp}\left(\phi_{\lambda}\right)=I\right\}} B_{0}^{K_{\lambda}}\left(\phi_{\lambda}\right)
$$

as 1 .

\section{$7 \quad$ No go theorems}

The main result of [AcLuVo99] was the existence of the Fock representation for the second order white noise. In [AcFrSk00] it was shown, among other things, that this representation can be interpreted as a representation of the current algebra over the Lie algebra $s l(2, \mathbb{R})$. The analogue representation 
for the first order white noise, which corresponds to the Heisenberg-Weyl algebra, had been known in physics for over 70 years.

Now: a current algebra over a Lie algebra is a functional version of the Lie algebra itself. More precisely it is an algebra of functions on some measure space $(X, \nu)$, (which in the case of $\left[\right.$ AcLuVo99] was $\mathbb{R}^{d}$ ) with values in this Lie algebra (such algebras were introduced and widely studied in the 1960's and, in the more recent mathematical literature, they are sometimes called "Kac-Moody algebras").

Now it might seem, at first glance, natural to conjecture that, if a Lie algebra has a Fock (lowest weight) representation, then the associated current algebra too have one. This is certainly true if the measure space $(X, \nu)$ has a finite number of points because in this case the current representation is a finite tensor product of the original one.

For example the Lie algebra generated by the Heisenberg-Weyl algebra and $\operatorname{sl}(2, \mathbb{R})$, called the Schrödinger algebra, has been widely studied in the literature and, since the Schrödinger representation exists in any finite dimension, the associated current algebra over $(X, \nu)$ has a Fock representation for any space $X$ with a finite number of points.

In the paper [Śnia99], devoted to the extension of the results of [AcLuVo99] to the free case, Śniady proved the following result.

Theorem The joint Fock representation of the first and second order white noise, i.e. of the Schrödinger algebra, cannot exist.

This theorem was generalized in [AcFrSk00] and further generalized by Accardi, Boukas and Franz [AcBouFr05] whose result, reported below, destroyed the hopes of a naive generalization, the higher powers of white noise, of the results obtained for the second power.

Theorem 1 In the notation (2), denote

$$
B_{k}^{n}:=\int_{\mathbb{R}^{d}} \chi_{I}(t) b_{t}^{\dagger} b_{t}^{k} d t
$$

where $\chi_{I}$ is the characteristic function of the interval $I \subseteq \mathbb{R}$ (taking value 1 on $I$ and 0 elsewhere). Let $\mathcal{L}$ be a Lie $*$-algebra with the following properties:

(i) $\mathcal{L}$ contains $B_{0}^{n}$, and $B_{0}^{2 n}$

(ii) the $B_{K}^{N}$ satisfy the higher power commutation relations . 
Then $\mathcal{L}$ does not have a Fock representation if the interval $I$ is such that

$$
\mu(I) \leq \frac{1}{c}
$$

where $c$ denotes the renormalization constant.

This theorem means that we cannot hope to have a single representation including all the higher powers of white noise: the best one can hope is to form, for each $n$, the smallest Lie algebra generated by $B_{0}^{n}$ and $B_{n}^{0}$ and look for a representation of it.

The difficulty with this programme is that, as soon as $n \geq 3$ these Lie algebras are infinite dimensional and not so widely studied. In particular one cannot apply the general methods of [AcFrSke00], which heavily used the known theory of irreducible unitary representations of $\operatorname{sl}(2, \mathbb{R})$, and one has to go back to the direct method of [AcLuVo99] which however, in these cases is much more complex due to the more complex structure of the higher order commutation relations.

At the moment we do not know if such a representation exists even in the case $n=3$.

The following considerations show that this difficulty is related to and old open problem of classical probability.

In the case of $2-\mathrm{d}$ order noise and of higher orders with a single renormalization constant, the current algebra restricted to a single block Lie-span $\left\{B_{k}^{h}\left(\chi_{[0,1]}\right)\right\}$ is isomorphic to the 1 -mode Lie algebra

$$
\text { Lie- } \operatorname{span}-\left\{a^{+h} a^{k}\right\}
$$

Lemma 1 Let $\left(b_{t}^{ \pm}\right)$be the Boson Fock scalar white noise.

Suppose that the $k$-th power of white noise exists for some natural integer $k$ and admit a Fock representation. then the process

$$
\left\{W_{[s, t]}^{k}, \Phi,-\infty<s<t<+\infty\right\}
$$

defined formally by some renormalization of

$$
W_{[s, t]}^{k}=\int_{s}^{t}\left(b_{u}^{+}+b_{u}\right)^{k} d u
$$

should be a stationary additive independent increment process on $\mathbb{R}$. 
Lemma 2 The map

$$
a^{k} \rightarrow \int_{0}^{1} b_{t}^{k} d t=: B_{[0,1]}^{(k)}
$$

with the [AcBouFr05] renormalization, is a Lie algebra isomorphism.

Corollary. The Fock statistics of $\left(B_{[0,1]}^{(k)}\right)$ is the same as that of $\left(a_{k}\right)$.

Proof. This statistics is uniquely determined by the Lie algebra structure.

Lemma 3 The vacuum distribution of

$$
\int_{0}^{1}\left(b_{t}^{+}+b_{t}\right)^{n} d t=\int_{0}^{1} w_{t}^{n} d t
$$

coincides with that of

$$
\left(a^{+}+a\right)^{n}
$$

Proof. The statistics is uniquely determined by the Lie algebra structure

Corollary. If the Fock representation of the $n$-th power of white noise exists, then the vacuum distribution of

$$
\left(a^{+}+a\right)^{n}
$$

must be infinitely divisible.

Proof. ¿From Lemma (3) it follows that the distribution of $\left(a^{+}+a\right)^{n}$ is the same as the distribution of $\int_{0}^{1} d t\left(b_{t}^{+}+b_{t}\right)^{n}$ and from Lemma (1) we know that this is infinitely divisible.

Theorem 2 A necessary condition for the existence of the $n$-th power of white noise, renormalized as in [AcBouFr05] is that the $n$-th power of a classical Gaussian random variable is infinitely divisible.

In classical probability the $n$-th powers of the standard (1-dimensional, mean 0, variance 1) Gaussian random variable and their distributions have been widely studied. In particular it is known that, $\forall k \geq 1, \gamma^{2 k}$ is infinitely divisible.

However it is not known if, $\forall k \geq 1, \gamma^{2 k+1}$ is infinitely divisible.

This suggests the conjecture that the above mentioned programme might be realizable if one starts from even powers (which fortunately are closed under Lie brackets). 


\section{Appendix: Meixner's classification theorem}

\section{Orthogonal generating functions}

The purpose of this appendix is to give an exposition of the problem studied by Meixner and of its method of solution. To this goal we begin with some general definitions.

Definition 1 Let $\mu$ be a probability measure on $\mathbb{R}$ with moments of any order and let $P_{n}(x)(x \in \mathbb{R} ; n \in \mathbb{N})$, denote the orthogonal polynomials of $\mu$ normalized so that

$$
P_{0}(x) \equiv 1 ; \quad \text { leading term of } P_{n}(x)=1
$$

A function

$$
F: \mathbb{R} \times \mathbb{R} \rightarrow \mathbb{R}
$$

is called an orthogonal generating function if

$$
F(x, t)=\sum_{n} P_{n}(x) \frac{t^{n}}{n !}
$$

where the series in (6) converges weakly in $L^{2}(\mathbb{R}, \mu)$.

Problem. Under which conditions is a function $F: \mathbb{R} \times \mathbb{R}$ the orthogonal generating function of some probability measure on $\mathbb{R}$ ?

It is easy $t_{0}$ verify that a necessary condition (??) is that, denoting $\langle\cdot, \cdot\rangle$ the scalar product in $L^{2}(\mathbb{R}, \mu)$, one has

$$
\langle 1, F(\cdot, t)\rangle=\left\langle P_{0}, F(\cdot, t)\right\rangle=1
$$

In other words

$$
\int_{\mathbb{R}} F(x, t) \mu(d x)=1 ; \quad \forall t
$$

Theorem 3 Now suppose that condition (7) is satisfied with a function $F(x, t)$ of the special form

$$
F(x, t)=e^{x u(t)} f(t)
$$

where $u: \mathbb{R} \rightarrow \mathbb{R}$ is an invertible function such that 


$$
u(0)=0
$$

and $f$ is a function such that

$$
\begin{aligned}
& f(0)=1 \\
& u^{\prime}(0)=1
\end{aligned}
$$

If one assumes that $u$ is invertible, then the Laplace transform of $\mu$ is uniquely determined in its domain, by the formula

$$
\int_{\mathbb{R}} e^{x \tau} \mu(d x)=: \tilde{\mu}(\tau)=\frac{1}{f\left(u^{-1}(\tau)\right)}
$$

Proof. Then (7) becomes

$$
\int_{\mathbb{R}} e^{x u(t)} f(t) \mu(d x)=1
$$

Introducing the change of variable

$$
u(t)=: \tau ; \quad t=u^{-1}(\tau)
$$

the identity (15) or equivalently

$$
\int_{\mathbb{R}} e^{x u(t)} \mu(d x)=\frac{1}{f(t)}
$$

becomes (12).

Remark. The meaning of Theorem (3) is that a probability measure $\mu$, satisfying (7) and (8), is uniquely determined by the pair $(f, u)$ provided that $u$ is invertible.

In his paper [Meix34] Meixner:

(i) determines all pairs of functions $(f, u)$ satisfying conditions (7) and (8) for some probability measure $\mu$

(ii) shows that for each such pair $(f, u), u$ is invertible

(iii) explicitly determines all the corresponding probability measure. 
This justifies the following

Definition 2 A probability measure $\mu$ on $\mathbb{R}$ is called a Meixner measure if

(i) $\mu$ admits an orthogonal generating function $F(x, t)$

(ii) $F(x, t)$ has the form (8) for some pair of functions $(f, u)$ called the associated pair.

Finally let us prove that the ansatz (8) is coherent, i.e. that the series expansion of its right hand side has the form (6) with the $P_{m}(x)$ satisfying condition (4). This follows from the following:

Lemma 4 Let $f(t)$ be a formal power series with constant term

$$
f(0)=1
$$

and let $u(t)$ be a formal power series with constant term $u(0)=0$ and with linear term coefficient $u_{1}=1$, i.e.

$$
u(t)=t(1+[t]+\ldots)
$$

Then there exist polynomials $P_{n}(x)$, with leading coefficient equal to 1 ,

$$
P_{u}(x)=x^{n}+a_{n, 1} x^{n-1}+\cdots+a_{n, n}
$$

such that the following formal expansion holds

$$
f(t) e^{x u(t)}=\sum_{n=0}^{\infty} \frac{P_{n}(x)}{n !} t^{n}
$$

Proof. By assumption

$$
\begin{gathered}
f(t)=\sum_{n \geq 0} f_{n} t^{n} ; \quad f_{0}=1 \\
u(t)=\sum_{n \geq 0} u_{n} t^{n}=t+\tilde{u}_{2}(t) ; \quad u_{0}=0 ; \quad u_{1}=1
\end{gathered}
$$


We define the degree of a formal power series $\sum_{n>0} a_{n} x^{n}$, the smallest $n \in \mathbb{N}$ such that $a_{n} \neq 0$. For example, $f$ has degree $0, u$ has degree 1 and $\tilde{u}_{2}$ degree $\geq 2$. Moreover

$$
f(t) e^{x u(t)}=\sum_{n \geq 0} \frac{x^{n}}{n !} u(t)^{n} f(t)=\sum_{n \geq 0} \frac{x^{n}}{n !}\left[t+\tilde{u}_{2, n}(t)\right]^{n} f(t)
$$

Our assumption on $u$ implies that

$$
\begin{gathered}
u(t)^{n}=\left[t^{n}+\tilde{u}_{2 n}(t)\right] \\
\operatorname{deg} \tilde{u}_{2, n}(t) \geq n+1 \quad\left(u^{2 n}\right)
\end{gathered}
$$

and

$$
f(t)=[1+\tilde{f}(t)]
$$

with $\operatorname{deg} \tilde{f}(t) \geq 1$. Therefore

$u(t)^{n} f(t)=\left[t+\tilde{u}_{2}(t)\right]^{n}[1+\tilde{f}(t)]=\left[t^{n}+\tilde{u}_{2, n}(t)\right][1+\tilde{f}(t)]=t^{n}+\tilde{u}_{2, n}+t^{n} \tilde{f}+\tilde{u}_{2, n} \tilde{f}$

with

$$
\operatorname{deg} t^{n} \tilde{f} \geq n+1 ; \quad \operatorname{deg} \tilde{u}_{2, n} \tilde{f} \geq 2(n+1)
$$

therefore

$$
u(t)^{n} f(t)=t^{n}+B_{n}(t)
$$

with $\operatorname{deg} B_{n}(t) \geq n+1$. Therefore

$$
f(t) e^{x u(t)}=\sum_{k=0}^{n-1} \frac{x^{k}}{k !} u(t)^{k} f(t)+x^{n} \frac{t^{n}}{n !} \mid,+x^{n} \frac{B_{n}(t)}{n !}+C_{n+1}(t)
$$

with $\operatorname{deg} C_{n+1}(t) \geq n+1$. Therefore

$$
P_{n}(x)=\left.\partial_{t}^{n}\right|_{t=0} f(t) e^{x u(t)}=\sum_{k=0}^{n-1} \frac{x^{k}}{k !} c_{k}+x^{n}
$$

which proves that the leading coefficient of $P_{n}(x)$ is equal to 1 . 


\section{The equations for $f$ and for $v=u^{-1}$}

Denote $v$ the inverse formal power series of $u$, i.e. by definition

$$
u(v(t))=v(u(t))=t
$$

and denote

$$
D:=\frac{d}{d x}
$$

Then the following identity is clearly satisfied:

$$
v(D) e^{x u(t)} f(t)=v(u(t)) e^{x u(t)} f(t)=t e^{x u(t)} f(t)
$$

Taking $\partial_{t}^{n}$ of both sides of (17) one finds

$$
v(D) \partial_{t}^{n} e^{x u(t)} f(t)=\partial_{t}^{n}\left(t e^{x u(t)} f(t)\right)=t \partial_{t}^{n} e^{x u(t)} f(t)+n \partial_{t}^{n-1} e^{x u(t)} f(t)
$$

evaluating this at $t=0$ and keeping (17) into account, one finds

$$
v(D) P_{n}(x)=n P_{n-1}(x)
$$

On the other hand the $P_{n}(x)$ are the orthogonal polynomials of some measure $\psi$ on $\mathbb{R}$ if and only if there exist two sequences $\left(l_{n}\right)$ and $\left(k_{n}\right)$ of real numbers such that

$$
k_{n} \leq 0 ; \quad \forall n
$$

and

$$
P_{n+1}(x)=\left(x+l_{n+1}\right) P_{n}(x)+k_{n+1} P_{n-1}(x)
$$

with the convection that

$$
P_{-1}(x)=0
$$

Denoting $x$ the multiplication by $x$ and using the identity

$$
[v(D), x]=v^{\prime}(D)
$$

we find, combining (18) and (19):

$v(D) P_{n+1}(x)=(n+1) P_{n}(x)=\left(x+l_{n+1}\right) n P_{n-1}(x)+v^{\prime}(D) P_{n}(x)+k_{n+1}(n-1) P_{n-2}(x)$

while the usual Jacobi relation (19) is

$$
n P_{n}(x)=\left(x+l_{n}\right) n P_{n-1}(x)+n k_{n} P_{n-2}
$$


Subtracting (21) from (20) one finds

$$
P_{n}(x)=\left(l_{n+1}-l_{n}\right) n P_{n-1}(x)+v^{\prime}(D) P_{n}(x)+\left(\frac{k_{n+1}}{n}-\frac{k_{n}}{n-1}\right) n(n-1) P_{n-2}
$$

or equivalently

$$
\left(1-v^{\prime}(D)\right) P_{n}=\left(l_{n+1}-l_{n}\right) n P_{n-1}+\left(\frac{k_{n+1}}{n}-\frac{k_{n}}{n-1}\right) n(n-1) P_{n-2}
$$

Replacing $n$ by $n+1$ we find

$$
\left(1-v^{\prime}(D)\right) P_{n+1}=\left(l_{n+2}-l_{n+1}\right)(n+1) P_{n}+\left(\frac{k_{n+2}}{n+1}-\frac{k_{n+1}}{n}\right) n(n+1) P_{n-1}
$$

Applying $v(D)$ to both sides and dividing by $n+1$ one obtains

$$
\left(1-v^{\prime}(D)\right) P_{n}=\left(l_{n+2}-l_{n+1}\right) n P_{n-1}+\left(\frac{k_{n+2}}{n+1}-\frac{k_{n+1}}{n}\right) n(n-1) P_{n-2}
$$

Now, $P_{n}(x)$ cannot be identically zero because its leading coefficient is equal to 1 . Therefore comparing (22) and (23) we conclude that there exist constants $\lambda, \kappa$ such that

$$
\begin{gathered}
l_{n+1}-l_{n}=\lambda \Leftrightarrow l_{n+1}=n \lambda+1 \\
\frac{k_{n+1}}{n}-\frac{k_{n}}{n-1}=\kappa \Leftrightarrow k_{n+1}=n\left((n-1) \kappa+k_{2}\right)
\end{gathered}
$$

Notice that, since the $k_{n}$ are all negative, also $\kappa$ must be negative.

Moreover, given (24) and (25), (19) becomes:

$$
P_{n+1}(x)=\left(x+l_{1}+n \lambda\right) P_{n}(x)+n\left(k_{2}+(n-1) \kappa\right) P_{n-1}(x)
$$

We know that $k_{2} \leq 0$ and it cannot be $=0$, otherwise $\psi$ is a multiple of a $\delta$-measure, hence in (16) $P_{n}(x)=0, \forall n>1$ and, since $P_{0}(x)=1$ by assumption, equation (16) becomes

$$
f(t)=e^{-x u(t)}
$$

which can be satisfied for any $x, t \in \mathbb{R}$ if and only if

$$
u(t) \equiv 0, \quad f(t) \equiv 1
$$


which corresponds to a trivial solution. Thus for all non trivial solutions one must have

$$
k_{2}<0
$$

Since

$$
f(t)=\sum_{n \geq 0} \frac{P_{n}(0)}{n !} t^{n}
$$

it follows that

$$
\begin{gathered}
f^{\prime}(t)=\sum_{n \geq 1} P_{n}(0) \frac{t^{n-1}}{(n-1) !}=\sum_{n \geq 0} P_{n+1}(0) \frac{t^{n}}{n !} \\
=\sum_{n \geq 0}\left(l_{1}+n \lambda\right) P_{n}(0) \frac{t^{n}}{n !}+\sum_{n \geq 1} P_{n-1}(0)\left(k_{2}+(n-1) \kappa\right) \frac{t^{n}}{(n-1) !} \\
\sum_{n \geq 0} n \lambda P_{n}(0) \frac{t^{n}}{n !}=\lambda t \sum_{n \geq 1} P_{n}(0) \frac{t^{n-1}}{(n-1) !}=\lambda t f^{\prime}(t) \\
\sum_{n \geq 1} k_{2} P_{n-1}(0) \frac{t^{n}}{(n-1) !}=k_{2} t \sum_{n \geq 1} P_{n-1}(0) \frac{t^{n-1}}{(n-1) !}=k_{2} t \sum_{n \geq 0} P_{n}(0) \frac{t^{n}}{n !} \\
=k_{2} t f(t) \\
\kappa \sum_{n \geq 0} n(n-1) P_{n-1}(0) \frac{t^{n}}{n !}=\kappa t^{2} \sum_{n \geq 2} P_{n-1}(0) \frac{t^{n-2}}{(n-2) !}=\kappa t^{2} \sum_{n \geq 1} P_{n}(0) \frac{t^{n-1}}{(n-1) !} \\
=\kappa t^{2} f^{\prime}(t)
\end{gathered}
$$

¿From these identities one deduces that

$$
f^{\prime}(t)=l_{1} f(t)+\lambda t f^{\prime}(t)+k_{2} t f(t)+\kappa t^{2} f^{\prime}(t)
$$

or equivalently $f$ satisfies the equation

$$
\frac{f^{\prime}(t)}{f(t)}=\frac{l_{1}+k_{2} t}{1-\lambda t-\kappa t^{2}}
$$

Moreover from (18) and (22) we find

$$
\left(1-v^{\prime}(D)\right) P_{n}(x)=\lambda v(D) P_{n}(x)+\kappa v(D)^{2} P_{n}(x) ; \quad \forall n
$$


Therefore, as operators on $L_{\text {pol }}^{2}(\mathbb{R}, \mu)$

$$
1-v^{\prime}(D)=\lambda v(D)+\kappa v(D)^{2}
$$

or equivalently

$$
v^{\prime}=1-\lambda v-\kappa v^{2}
$$

Therefore the pair $(f, v)$ (equivalently $(f, u)$ ) is uniquely determined by the solutions of the equations (26), (28) respectively. Notice that the same polynomial

$$
1-\lambda t-\kappa t^{2}
$$

appears in both equations. According to the various possible values of the parameters $\lambda$, $\kappa$, we distinguish 5 possibilities:

(I) $\lambda=\kappa=0((29)$ has degree 0$)$

(II) $\kappa=0 ; \lambda \neq 0((29)$ has degree 1$)$

(III) $\lambda^{2}=-4 \kappa \neq 0(29)$ has degree 2 and one non zero root of multiplicity 2)

(IV) $\lambda^{2}>-4 \kappa>0(29)$ has degree 2 and 2 distinct non zero real roots)

(V) $0<\lambda^{2}<-4 \kappa(29)$ has degree 2 and 2 non zero complex conjugate roots)

The five Meixner classes are defined by $t$ the solutions of equations (28), (26) corresponding to the values of the parameters $(\lambda, \kappa)$ in the classes defined above.

Remark. In fact Meixner ([Meix34], Section 6) calls class (II) what we have called class (III) and conversely.

Moreover Meixner does not classify his five classes in terms of the parameters $(\lambda, \kappa)$ but in terms of two auxiliary parameters $(\alpha, \beta)$, related to $(\lambda, \kappa)$ by the equations

$$
\begin{aligned}
& \alpha+\beta=\lambda \\
& \alpha \beta=-\kappa
\end{aligned}
$$

In the following section we will describe the translation code between our parametrization and Meixner's. 


\section{Meixner's parametrization}

Theorem 4 (i) For any real numbers $\lambda, \kappa$ there exist complex numbers $\alpha, \beta$ such that the following identity holds

$$
1-\lambda t-\kappa t^{2}=(1-\alpha t)(1-\beta t)
$$

or equivalently

$$
\begin{gathered}
\alpha+\beta=\lambda \\
\alpha \beta=-\kappa
\end{gathered}
$$

(ii) The pair $(\lambda, \kappa)$ uniquely determines the pair $(\alpha, \beta)$ up to the permutation

$$
(\alpha, \beta) \rightarrow(\beta, \alpha)
$$

(iii) If in addition

$$
\kappa \leq 0
$$

then there are only four possibilities

$$
\begin{gathered}
\lambda=\kappa=0 \Leftrightarrow \alpha=\beta=0 \\
\kappa=0 ; \quad \lambda \neq 0 \Leftrightarrow(\alpha, \beta)=(\lambda, 0) \\
\kappa \neq 0 ; \quad \lambda=0 \Leftrightarrow(\alpha, \beta)=\left(-i|\kappa|^{1 / 2}, i|\kappa|^{1 / 2}\right) \\
\kappa \neq 0 ; \lambda \neq 0 \Leftrightarrow(\alpha, \beta)=\left(\frac{\lambda}{2}-\sqrt{\frac{\lambda^{2}}{4}+\kappa,} \frac{\lambda}{2}+\sqrt{\frac{\lambda^{2}}{4}+\kappa}\right)=:\left(t_{-}, t_{+}\right)
\end{gathered}
$$

where, in all the above identities $(\alpha, \beta)$ has been identified to $(\beta, \alpha)$ and the square roots are the positive ones.

Moreover the last possibility (37) splits into three according to the following situations:

$$
\frac{\lambda^{2}}{4|\kappa|^{2}}=\frac{1}{|\kappa|} \Leftrightarrow \frac{\lambda^{2}}{4}=|\kappa|
$$

i.e. only one real solution

$$
\frac{\lambda^{2}}{4}>|\kappa|
$$

i.e. two distinct real solutions

$$
\frac{\lambda^{2}}{4}<|\kappa|
$$

i.e. two complex conjugate solutions. 
(iv) The five Meixner classes are characterized by the following values of the $\operatorname{pair}(\lambda, \kappa)$ :

(I) $\lambda=\kappa=0$

(II) $\lambda, \kappa \neq 0, \lambda= \pm 2|\kappa|^{1 / 2}$

(III) $\kappa=0 ; \lambda \neq 0$

$(I V) \lambda, \kappa \neq 0 ; \lambda^{2}>4|\kappa|$

(V) $\lambda, \kappa \neq 0 ; \lambda^{2}<4|\kappa|$.

Let us first discuss the equation

$$
0=1-\lambda t-\kappa t^{2}
$$

If

$$
\lambda=\kappa=0
$$

there are no solutions.

If

$$
\kappa=0 ; \lambda \neq 0
$$

there is only one solution

$$
t_{\kappa_{0}}=\frac{1}{\lambda}
$$

If

$$
\kappa \neq 0 ; \lambda=0
$$

then (41) becomes

$$
0=1-\kappa t^{2}=1+|\kappa| t^{2}
$$

which has only 2 purely imaginary complex conjugate solutions:

$$
t_{\lambda_{0}, \pm}= \pm i \sqrt{\frac{1}{|\kappa|}}
$$

If both

$$
\kappa, \lambda \neq 0
$$

then equation (41) can be written

$$
0=|\kappa| t^{2}-\lambda t+1 \Leftrightarrow 0=t^{2}-\frac{\lambda}{|\kappa|} t+\frac{1}{|\kappa|}=\left(t-\frac{\lambda}{2|\kappa|}\right)^{2}-\frac{\lambda^{2}}{4|\kappa|^{2}}+\frac{1}{|\kappa|}
$$


which has exactly one solution if and only if (38) holds.

In this case the solution is

$$
t_{1 s}=\frac{\lambda}{2|\kappa|}
$$

and, due to the relation (38) there are 2 possibilities

$$
\frac{\lambda}{2}= \pm|\kappa|^{1 / 2}
$$

giving rise to the solutions

$$
t_{1 s, \pm}= \pm \frac{1}{|\kappa|^{1 / 2}}
$$

The two remaining possibilities, beyond (38) are (39) and (40).

Condition (39) corresponds to two distinct real solutions

$$
t_{ \pm}=\frac{\lambda}{2|\kappa|} \pm \frac{\lambda^{2}}{4|\kappa|^{2}}-\frac{1}{|\kappa|}=\frac{1}{|\kappa|}\left(\frac{\lambda}{2} \pm \sqrt{\frac{\lambda^{2}}{4}-|\kappa|}\right)
$$

Condition (40) corresponds to two complex conjugate solutions

$$
s_{ \pm}=\frac{1}{|\kappa|}\left(\frac{\lambda}{2} \pm i \sqrt{|\kappa|-\frac{\lambda^{2}}{4}}\right)
$$

Now let us consider the identity (30) which is equivalent to

$$
1-\lambda t-\kappa t^{2}=1-(\alpha+\beta) t+\alpha \beta t^{2}
$$

It is clear that the pair $(\alpha, \beta)$ is a solution if and only if the pair $(\beta, \alpha)$ is.

Equating coefficients we find (31), (32).

¿From these we deduce

$$
-\kappa=(\lambda-\beta) \beta=\lambda \beta-\beta^{2}
$$

i.e.

$$
\beta^{2}-\lambda \beta-\kappa=0 \leftrightarrow\left(\beta-\frac{\lambda}{2}\right)^{2}-\frac{\lambda^{2}}{4}-\kappa=0 \Leftrightarrow \beta-\frac{\lambda}{2}= \pm \sqrt{\frac{\lambda^{2}}{4}+\kappa}
$$


This gives the solutions

$$
\begin{aligned}
& \beta_{ \pm}=\frac{\lambda}{2} \pm \sqrt{\frac{\lambda^{2}}{4}+\kappa} \\
& \alpha_{ \pm}=\frac{\lambda}{2} \mp \sqrt{\frac{\lambda^{2}}{4}+\kappa}
\end{aligned}
$$

which satisfy the condition

$$
\left(\beta_{+}, \alpha_{+}\right)=\left(\alpha_{-}, \beta_{-}\right)
$$

Let us discuss the possible solutions of the system (32), (31) corresponding to the various possibilities for the parameters $\lambda$ and $\kappa$.

(34) is obvious.

Clearly (42) holds if and only if

$$
\alpha=\beta=0
$$

Now suppose that (44) holds then

$$
\begin{aligned}
& \beta_{+}=\lambda \neq 0 ; \quad \beta_{-}=0 \\
& \alpha_{+}=0 ; \quad \alpha_{-}=\lambda \neq 0
\end{aligned}
$$

that is, exactly one number, in the pair $(\alpha, \beta)$ is $\neq 0$.

Conversely, if this is the case, then $\lambda$ must be $\neq 0$, otherwise

$$
\alpha_{ \pm}=-\beta_{ \pm}
$$

and it is impossible that exactly one is $\neq 0$. Moreover this condition can be fulfilled only if $\lambda^{2} / 4+\kappa$ is real.

In this case one has always

$$
\beta_{+}>0 ; \quad \alpha_{-}>0
$$

Thus the condition that exactly one in the pair $(\alpha, \beta)$ is different from zero can be fulfilled only if either

$$
\alpha_{+}=\frac{\lambda}{2}-\sqrt{\frac{\lambda^{2}}{4}+\kappa}=0
$$


or

$$
\beta_{-}=\frac{\lambda}{2}-\sqrt{\frac{\lambda^{2}}{4}+\kappa}=0
$$

Thus the two conditions coincide and are both equivalent to

$$
\kappa=0
$$

This proves (35).

Now suppose that condition (44) holds. Then the system (32), (31) becomes

$$
\begin{gathered}
\alpha=-\beta \\
\kappa=\beta^{2}
\end{gathered}
$$

Since $\kappa \neq 0$, this means that $\beta$ must be purely imaginary and $\neq 0$. Conversely, if this is the case and (51) holds, then (45) holds. This proves (36).

If condition (45) holds, then the system (32), (31) has 2 distinct solutions satisfying

$$
\left(\alpha_{+}, \beta_{+}\right),\left(\alpha_{-}, \beta_{-}\right)=\left(\beta_{+}, \alpha_{+}\right)
$$

Conversely, if this is the case, then (45) must hold because, if either $\lambda$ or $\kappa$ are zero, then (53) cannot define two distinct solutions.

Finally note that the above discussion is valid in both cases when the solutions of (41) are real or complex, i.e. if either condition (39) or (40) hold.

This proves (37).

This completes the proof of (iii).

The 1-st Meixner class is clearly characterized by the condition

$$
\lambda=\kappa=0
$$

The condition characterizing the 2-d Meixner class is equivalent to the case (37) under condition (38), i.e. when equation (41) has a unique non zero real solution.

The 3-d Meixner class is equivalent to the case (35).

The 4-th Meixner class is equivalent to the case (37) under the condition (39), corresponding to two distinct real nonzero solutions.

The 5-th Meixner class is equivalent to the case (37) under the condition (40), corresponding to two complex conjugate solutions. 


\section{Solutions of the equation for $v$}

In the present section we discuss the solutions of equation (28) corresponding to the various Meixner classes.

Class I: $\lambda=\kappa=0$. In this case equation (28) becomes

$$
v^{\prime}=1
$$

By assumption

$$
u(t)=t+t^{2} u_{2}(t)
$$

where $u_{2}(t)$ is an arbitrary formal power series. Moreover

$$
t=v(u(t))=v_{0}+v_{1} u(t)+\sum_{n \geq 2} v_{n} u(t)^{n}
$$

Thus

$$
0=v(0)=v_{0}
$$

and the unique solution of (54) with initial condition (55) is

$$
v(\tau)=\tau
$$

Class II: $\lambda, \kappa \neq 0, \lambda= \pm 2|\kappa|^{1 / 2}$. In this case equation (28) becomes

$$
v^{\prime}(\tau)=1 \mp 2|\kappa|^{1 / 2} v(\tau)+v(\tau)^{2}|\kappa|=\left(1 \mp|\kappa|^{1 / 2} v(\tau)\right)^{2}
$$

This gives

$$
t+c=\int \frac{d v}{\left(1 \mp|\kappa|^{1 / 2} v\right)^{2}}=: I
$$

which is of the form

$$
\int \frac{a+b t}{c t \mp 1)^{2}} d t
$$

with

$$
b=0 ; \quad a=1 ; \quad c=|\kappa|^{1 / 2}
$$

Therefore the solution is

$$
I=-\frac{|\kappa|^{-1 / 2}}{|\kappa|^{1 / 2} v \mp 1}=\frac{1}{|\kappa| v \pm|\kappa|^{1 / 2}}
$$


This gives

$$
t+c=\frac{1}{|\kappa| v \pm|\kappa|^{1 / 2}}
$$

or

$$
\frac{1}{(t+c)|\kappa|} \mp \frac{1}{|\kappa|^{1 / 2}}=v(t)
$$

and condition (55) is satisfied if and only if

$$
c= \pm \frac{1}{|\kappa|^{1 / 2}}
$$

This gives

$$
\begin{gathered}
v(t)=\left(\frac{1}{\left(t \pm|\kappa|^{-1 / 2}\right)|\kappa|} p \frac{1}{|\kappa|^{1 / 2}}\right)=\frac{1}{|\kappa| t \pm|\kappa|^{1 / 2}} \mp \frac{1}{|\kappa|^{1 / 2}} \\
=\frac{|\kappa|^{1 / 2} \mp\left(|\kappa| t \pm|\kappa|^{1 / 2}\right)}{|\kappa|^{1 / 2}\left(|\kappa| t \pm|\kappa|^{1 / 2}\right)}=\frac{\mp|\kappa|^{1 / 2} t}{|\kappa|^{1 / 2}\left(|\kappa| t \pm|\kappa|^{1 / 2}\right)}=\mp \frac{t}{|\kappa| t \pm|\kappa|^{1 / 2}}
\end{gathered}
$$

Class III: $\kappa=0 ; \lambda \neq 0$. In this case equation (28) becomes

$$
v^{\prime}=1-\lambda v \Leftrightarrow \int \frac{d v}{1-\lambda v}=t+c
$$

Thus

$$
\begin{gathered}
t+c=-\frac{1}{\lambda} \ln (1-\lambda v) \\
e^{t} e^{c}=(1-\lambda v)^{-1 / \lambda} \Leftrightarrow e^{-\lambda t} e^{-\lambda c}=1-\lambda v \\
v=\frac{1}{\lambda}\left(1-e^{-\lambda t} e^{-\lambda c}\right)
\end{gathered}
$$

The condition

$$
v(0)=0
$$

fixes $c=0$, so that

$$
v(\tau)=\frac{1}{\lambda}\left(1-e^{-\lambda \tau}\right)
$$

Class IV: $\lambda, \kappa \neq 0 ; \lambda^{2}>4|\kappa|$

In this case equation (28) becomes

$$
v^{\prime}=|\kappa|\left(v^{2}-\lambda|\kappa| v+\frac{1}{|\kappa|}\right)=|\kappa|\left(v-t_{+}\right)\left(v-t_{-}\right)
$$


or equivalently

$$
|\kappa| t+c=\int \frac{d v}{\left(v-t_{+}\right)\left(v-t_{-}\right)}=\ln \left[\frac{\left(v-t_{+}\right)}{\left(v-t_{-}\right)}\right]^{\left(t_{+}+t_{-}\right)^{-1}}
$$

with

$$
\left(t_{+}+t_{-}\right)^{-1}=\frac{|\kappa|}{\lambda}
$$

This gives

$$
\begin{gathered}
|\kappa| t+c=\ln \left(\frac{v-t_{+}}{v-t_{-}}\right)^{|\kappa| / \lambda} \\
e^{|\kappa| t+c}=\left(\frac{v-t_{+}}{v-t_{-}}\right)^{|\kappa| / \lambda} \\
e^{\lambda t} e^{\lambda c /|\kappa|}=\frac{v-t_{+}}{v-t_{-}}
\end{gathered}
$$

and the condition $v(0)=0$ fixes

$$
\begin{gathered}
e^{\lambda c /|\kappa|}=\frac{t_{+}}{t_{-}} \\
e^{\lambda t} t_{+}\left(v-t_{-}\right)=t_{-}\left(v-t_{+}\right) \Leftrightarrow\left(e^{\lambda t} t_{+}-t_{-}\right) v=e^{\lambda t} t_{+} t_{-}-t_{+} t_{-} \\
v=\frac{e^{\lambda t}-1}{e^{\lambda t} t_{+}-t_{-}} t_{+} t_{-}=\frac{1}{|\kappa|} \frac{e^{\lambda t}-1}{e^{\lambda t} t_{+}-t_{-}}
\end{gathered}
$$

In conclusion

$$
v(\tau)=\frac{e^{\lambda \tau}-1}{e^{\lambda \tau} \tau_{+}-\tau_{-}}
$$

where

$$
\tau_{ \pm}:=\frac{\lambda}{2}\left(1 \pm \sqrt{1-\frac{4|\kappa|}{\lambda^{2}}}\right)
$$

Class V: $\lambda, \kappa \neq 0 ; \lambda^{2}<4|\kappa|$

The result is the same as in the case of Class IV, i.e. (59). 


\section{Solutions of the equation for $f$}

\section{Class I}

$$
\lambda=\kappa=0
$$

In this case

$$
\frac{f^{\prime}(t)}{f(t)}=l_{1}+k_{2} t \Leftrightarrow f(t)=e^{l_{1} t+\frac{1}{2} k_{2} t^{2}}
$$

and the condition

$$
f(0)=1
$$

is satisfied.

\section{Class II}

Let us consider the case

$$
\begin{gathered}
\kappa \neq 0 \\
\lambda^{2}=4|\kappa|
\end{gathered}
$$

In this case we have

$$
\begin{gathered}
\ln f(t)=\int \frac{l_{1}+k_{2} t}{\left(|\kappa|^{1 / 2} t \mp 1\right)^{2}} d t=\log \left(|\kappa|^{1 / 2} t \mp 1\right)^{k_{2}|\kappa|}\left(-\frac{l_{1}}{|\kappa|^{1 / 2}} \mp \frac{k_{2}}{|\kappa|}\right) \frac{1}{|\kappa|^{1 / 2} t \mp 1} \\
f(t)=\frac{\exp \left(-\frac{l_{1}}{|\kappa|^{1 / 2}} \mp \frac{k_{2}}{|\kappa|}\right) \frac{1}{|\kappa|^{1 / 2}} t \mp 1}{\left(|\kappa|^{1 / 2} t \mp 1\right)^{\left|k_{2}\right| /|\kappa|}}
\end{gathered}
$$

\section{Class III}

If

$$
\lambda \neq 0 ; \quad \kappa=0
$$

then

$$
\partial \ln f(t)=\frac{l_{1} /\left|K_{2}\right|-t}{1 / \lambda-t} \cdot \frac{\left|k_{2}\right|}{\lambda}
$$

Therefore in case (64)

$$
\begin{aligned}
\ln f(t) & =\frac{\left|k_{2}\right|}{\lambda}\left(t+\ln \left(\frac{1}{\lambda}-t\right)^{\frac{1}{\lambda}-\frac{l_{1}}{\left|k_{2}\right|}}\right) \\
f(t) & =e^{t \frac{\left|k_{2}\right|}{\lambda}}\left(\frac{1}{\lambda}-t\right)^{\frac{\left|k_{2}\right|}{\lambda}\left(\frac{1}{\lambda}-\frac{l_{1}}{\left|k_{2}\right|}\right)}
\end{aligned}
$$




\section{Classes IV and V}

These classes are characterized by the condition

$$
\lambda^{2} \neq 4|\kappa| \neq 0
$$

(real roots, class IV; complex conjugate roots, class V). In this case the characteristic polynomial $1-\lambda t-\kappa t^{2}$ has 2 different roots $t_{ \pm}$such that

$$
|\kappa|^{1 / 2} t_{ \pm}=\frac{\lambda}{2|\kappa|^{1 / 2}} \pm \sqrt{\frac{\lambda^{2}}{4|\kappa|}-1}
$$

Therefore the solution of equation (26) is

$$
\ln f(t)=\frac{1}{|\kappa|} \frac{l_{1}+k_{2} t}{\left(t-t_{+}\right)\left(t-t_{-}\right)} d t=\int \frac{l_{1}+k_{2} t}{|\kappa| t^{2}-\lambda t+1} d t
$$

The solution of (67) satisfying

$$
0=\ln f(1)=\ln 1
$$

is given by

$$
\begin{gathered}
\ln f(t)=\ln \left(1-t / t_{+}\right)^{A}\left(1-t / t_{-}\right)^{B} \\
A=\frac{a+b t_{+}}{t_{+}-t_{-}}=\frac{1}{|\kappa|} \frac{l_{1}+k_{2} t_{+}}{t_{+}-t_{-}}=\frac{l_{1}+k_{2} t_{+}}{\sqrt{\lambda^{2}-4|\kappa|}} \\
B=-\frac{a+b t}{t_{+}-t_{-}}=-\frac{1}{|\kappa|} \frac{l_{1}+k_{2} t}{t_{+}-t_{-}}=-\frac{l_{1}+k_{2} t_{-}}{\sqrt{\lambda^{2}-4|\kappa|}}
\end{gathered}
$$

It is convenient to write

$$
\frac{e_{1}}{\sqrt{\lambda^{2}-4|\kappa|}}=: \lambda_{1} ; \quad \frac{k_{2}}{\sqrt{\lambda^{2}-4|\kappa|}}=: \kappa_{2}
$$

With these notations

$$
A=\lambda_{1}+\kappa_{2} t_{+} ; \quad B=-\left(\lambda_{1}+\kappa_{2} t_{-}\right)
$$

and (68) becomes equivalent to

$$
f(t)=\left[\frac{\left(1-t / t_{+}\right)}{\left(1-t / t_{-}\right)}\right]^{\lambda_{1}}\left[\frac{\left(1-t / t_{+}\right)^{t_{+}}}{\left(1-t / t_{-}\right)^{t_{-}}}\right]^{\kappa_{2}}
$$

Remark. In the paper p] (pg. 10) Meixner assumes that

$$
l_{1}=0
$$

therefore, due to (69), in order to recover his expression for $f(t)$, one has to put $\lambda_{1}=0$ in (71). 


\section{The equations for $u$}

Now notice that, if

$$
u(t)=\tau \Leftrightarrow u^{-1}(\tau)=t
$$

then

$$
t=u^{-1}(u(t))=v(u(t)) \Rightarrow 1=v^{\prime}(u(t)) u^{\prime}(t) \Leftrightarrow v^{\prime}(u(t))=\frac{1}{u^{\prime}(t)}
$$

Therefore if $v^{\prime}(\tau)=F(v(\tau))$ then

$$
\frac{1}{u^{\prime}(t)}=v^{\prime}(u(t))=F(v(u(t)))=F(t)
$$

and in our case this becomes

$$
\frac{1}{u^{\prime}(t)}=1-\lambda v(u(t))-\kappa v^{2}(u(t))=1-\lambda t-\kappa t^{2}
$$

or

$$
\begin{gathered}
u^{\prime}(t)=\frac{1}{1-\lambda-\kappa t^{2}} \\
u(t)=\int \frac{d t}{1-\lambda t-\kappa t^{2}}=\int \frac{d t}{|\kappa| t^{2}-\lambda t+1}
\end{gathered}
$$

Therefore in the case (61)

$$
u(t)=\int d t=t
$$

because $u(0)=0$.

In the case (64)

$$
u(t)=\int \frac{d t}{1-\lambda t} ; \quad \lambda \neq 0
$$

Therefore

$$
u(t)=\ln \frac{1}{(1-\lambda t)^{1 / \lambda}}
$$

In case (63), i.e.

$$
\begin{gathered}
\lambda= \pm 2|\kappa|^{1 / 2} \neq 0 \\
u(t)=\int \frac{d t}{\left(|\kappa|^{1 / 2}[ \pm 1)^{2}\right.}=-\frac{1}{|\kappa|^{1 / 2}}\left(|\kappa|^{1 / 2} t \pm 1\right)^{-1}
\end{gathered}
$$


In case (65) the polynomial (29) has two roots given by (66). Therefore

$$
\begin{gathered}
u(t)=\int \frac{d t}{|\kappa|\left(t-t_{+}\right)\left(t-t_{-}\right)}=\frac{1}{|\kappa|} \int \frac{d t}{t-t_{+}}-\frac{1}{|\kappa|} \int \frac{d t}{t-t_{-}} \\
=\frac{1}{|\kappa|} \ln \frac{t-t_{+}}{t-t_{-}}=\ln \left(\frac{t-t_{+}}{t-t_{-}}\right)^{1 /|\kappa|}
\end{gathered}
$$

I $\alpha=\beta=\lambda=\kappa=0$

$$
\alpha \psi(x)=e^{x^{2} / 2 k} d x
$$

Hermite's polynomials.

II $\alpha=\beta \neq 0$

$$
\begin{gathered}
\alpha>0 \psi^{\prime}(x)= \begin{cases}\left(-x-\frac{k_{2}}{\alpha}\right)^{-1-k_{2} / \alpha^{2}} e^{x / \alpha} & -\infty<x<-\frac{k_{2}}{\alpha} \\
0 & -\frac{k_{2}}{\alpha}<x<\infty\end{cases} \\
\alpha<0 \psi^{\prime}(x)= \begin{cases}0 & -\infty<x<-\frac{k_{2}}{\alpha} \\
\left(x+\frac{k_{2}}{\alpha}\right)-1-k_{2} / \alpha^{2} e^{x / \alpha} & -\frac{k_{2}}{\alpha}<x<\infty\end{cases}
\end{gathered}
$$

confluent hypergeometric polynomials

III $\alpha \neq 0, \beta=\kappa=0$

$$
\alpha \psi(x)=\sum_{n=0}^{\infty} \frac{1}{n !}\left(-\frac{k_{2}}{\alpha^{2}}\right)^{n} \delta_{-\frac{k_{2}}{\alpha}-\frac{\alpha}{n}}
$$

Charlier's polynomials

IV $\alpha \neq \beta, \kappa \neq 0, \alpha, \beta$ real

e.g. $|\alpha|>|\beta|$

$$
d \psi(x)=\sum_{n=0}^{\infty}\left(-\frac{\beta}{\alpha}\right)^{n}\left(\begin{array}{c}
k_{2} / \alpha \beta \\
n
\end{array}\right) \delta_{-\frac{k_{2}}{\alpha}-(\alpha-\beta) n}
$$

$\mathrm{V} \alpha \neq \beta, \kappa \neq 0 \bar{\alpha}=\beta$

$\exists \operatorname{Im} \alpha>0 \begin{gathered}\alpha=|\alpha| e^{i \varphi} \\ \beta=|\alpha| e^{-i \varphi}\end{gathered}$

$$
\begin{gathered}
\psi^{\prime}(x)=\left(-\frac{\beta}{\alpha}\right)^{x /(\beta-\alpha)} \Gamma\left(\frac{x}{\beta-\alpha}+\frac{k_{2}}{\beta(\beta-\alpha)}\right) \Gamma\left(\frac{x}{\alpha-\beta}+\frac{k_{2}}{\alpha(\alpha-\beta)}\right) \\
\left|\arg \left(-\frac{\beta}{\alpha}\right)\right|<\pi-\pi<\arg (-\beta / \alpha)<\pi
\end{gathered}
$$




\section{Moments of the Meixner measures}

In this section we derive a simple formula which expresses the moments of a Meixner measure in terms of the associated Meixner pair.

Taking $\partial_{t}$-derivatives of both sides of (15) one obtains

$$
\partial_{t} \int_{\mathbb{R}} e^{x u(t)} \mu(d x)=\int x u^{\prime}(t) e^{x u(t)} \mu(d x)=\partial_{t} \frac{1}{f(t)}
$$

or equivalently, writing

$$
\begin{gathered}
u^{(n)}(t):=\partial_{t} u^{(n-1)}(t) ; \quad u^{(0)}(t):=u(t) \\
\int_{\mathbb{R}} x e^{x u(t)} \mu(d x)=\frac{1}{u^{(1)}(t)} \partial_{t} \frac{1}{f(t)}
\end{gathered}
$$

In particular, putting $t=0$ in (73) and using (9) one finds the first moment of $\mu$, i.e.

$$
m_{1}(\mu)=\int_{\mathbb{R}} x \mu(d x)=\left.\frac{1}{u^{(1)}(0)} \partial_{t}\right|_{t=0} \frac{1}{f(t)}
$$

Taking derivatives of both sides of (73) one finds

$$
\int_{\mathbb{R}} x^{2} u^{\prime}(t) e^{x u(t)} \mu(d x)=\partial_{t} \frac{1}{u^{(1)}(t)} \partial_{t} \frac{1}{f(t)}
$$

or equivalently

$$
\int_{\mathbb{R}} x^{2} e^{x u(t)} \mu(d x)=\frac{1}{u^{(1)}(t)} \partial_{t} \frac{1}{u^{(1)}(t)} \partial_{t} \frac{1}{f(t)}
$$

Now, considering $u^{(1)}$ as a multiplication operator in $L^{2}(\mathbb{R}, \mu)$ and $\partial_{t}$ as an operator in the same space, one can introduce the notation

$$
\Lambda_{u}:=\frac{1}{u^{(1)}} \partial_{t}
$$

So that, if $\varphi$ is another multiplication operator in $L^{2}(\mathbb{R}, \mu)$ :

$$
\Lambda_{u} \varphi(t)=\frac{1}{u^{(1)}(t)}\left(\partial_{t} \varphi\right)(t)
$$

In these notations (75) becomes

$$
\int_{\mathbb{R}} x^{2} e^{x u(t)} \mu(d x)=\Lambda_{u}^{2} \frac{1}{f}(t)
$$


which gives the second moment of $\mu$ by evaluating (78) at $t=0$

$$
m_{2}(\mu)=\int_{\mathbb{R}} x^{2} \mu(d x)=\Lambda_{u}^{2} \frac{1}{f}(0)
$$

Now suppose by induction that,

$$
\int_{\mathbb{R}} x^{n} e^{x u(t)} \mu(d x)=\Lambda_{u}^{n} \frac{1}{f}(t)
$$

then, taking derivatives of both sides, one finds

$$
\int_{\mathbb{R}} x^{n+1} u^{\prime}(t) e^{x u(t)} \mu(d x)=\partial_{t} \Lambda_{u}^{n} \frac{1}{f}(t)
$$

or equivalently

$$
\int_{\mathbb{R}} x^{n+1} e^{x u(t)} \mu(d x)=\frac{1}{u^{(1)}(t)} \partial_{t} \Lambda_{u}^{n} \frac{1}{f}(t)=\Lambda_{u}^{n+1} \frac{1}{f}(t)
$$

and therefore (80) holds for each $n \in \mathbb{N}$. In particular, taking $t=0$ in (80) one finds the $n$-th moment of $u$ :

$$
m_{n}(\mu)=\int_{\mathbb{R}} x^{n} \mu(d x)=\Lambda_{u}^{n} \frac{1}{f}(0) ; \quad \forall n \in \mathbb{N}
$$

In other words:

Theorem 5 Suppose that a probability measure $\mu$ on $\mathbb{R}$ has an orthogonal generating function of the form (8) for a pair of $C^{\infty}$-functions $(u, f)$ from $\mathbb{R}$ to $\mathbb{R}$. Then $\mu$ is polynomially determined by the pair $(u, f)$ through formula (81).

\section{Bibliography}

[AcLuVo02] Accardi L., Lu Y.G., Volovich I.: Quantum Theory and its Stochastic Limit, Springer Verlag (2002)

[AcLuVo99] Accardi L., Lu Y.G., Volovich I.V.: White noise approach to classical and quantum stochastic calculi, Lecture Notes of the Volterra International School of the same title, Trento, Italy, 1999, Volterra Preprint N. 375 July (1999) 
[AcFrSk00] Accardi L., Franz U., Skeide M.: Renormalized squares of white noise and other non- Gaussian noises as Levy processes on real Lie algebras, Comm. Math. Phys. 228 (2002) 123-150 Preprint Volterra, N. 423 (2000)

Accardi L.: The white noise approach to stochastic calculus: motivations, applications, developments, in: Colloque SMT 2000, p. 1-18 Plenary Lecture given at: Colloque Société Mathématique de Tunisie, Sousse, 27-30 mars 2000

[AcBo97] Luigi Accardi, Marek Bożejko: Interacting Fock spaces and Gaussianization of probability measures Infinite dimensional analysis, quantum probability and related topics, 2, N. 4 (1998) Volterra Preprint (1997) N. 321

[AcKuSt02] Accardi L., Kuo H.-H., Stan A.: Orthogonal polynomials and interacting Fock spaces, Infinite Dimensional Anal. Quantum Probab. Related Topics (IDA-QP) 7 (4) (2004) 485-505

[AcBouFr05] Luigi Accardi, Andreas Boukas, Uwe Franz: Non existence of the Fock representation for the renormalized higher powers of white noise, to be submitted to IDA-QP (2005)

[AcAyOu03] Luigi Accardi, Wided Ayed, Habib Ouerdiane: White Noise Approach to Quantum Stochastic Calculus, talk given at the: COllOQUE INTERNATIONAL DE MATHEMATIQUES, ANALYSE ET PROBABILITES, 20-25 Octobre 2003, Hammamet, (Tunisie) to appear

[Ayed05] Wided Ayed: PhD thesis (to appear)

[BEREZb98] Yu.M. Berezansky: Commutative Jacobi fields in Fock space, J. Func. Anal. 138 (1998) 163-190.

[BEREZa97] Yu.M. Berezansky: On the direct and inverse spectral problem for Jacobi fields, Algebra i Analys (in russian) 9 (6) (1997), english transl. St. Petersburg Math. J. 9 (6) (1997).

[BEKO94] Yu.M. Berezansky, Yu.G. Kondratiev: Spectral Methods in infinite dimensional analysis, Kluwer (1994).

[BELILY95] Yu.M. Berezansky, V.O. Livinsy,, E.W. Lytvynov: A generalization of Gaussian white noise analysis, Methods Func. Anal. and Topol. 1 (1995) 28-55.

[BruRo91] F.T. Bruss, L.C. Rogers: Pascal processes and their characterization, Stochastic Process. Appl. 37 (1991) 331-338

[FeinScho93] P. Feinsilver, R. Schott: Algebraic Structures and Operator Calculus, Vol. I: Representations and Probability Theory, Kluwer Academic Publishers, Dordrecht (1993) 
[Grig01] B. Grigelionis: Generalized z-distributions and related stochastic processes, Lithuanian Math. J. 41 (2001) 303-319

[Grig99] B. Grigelionis: Processes of Meixner type, Lithuanian Math. J. 39 (1999) 33-41.

[Grig00c] B. Grigelionis: On generalized z-diffusions, Preprint Vilnius (2000).

[Hida01] Hida T.: Selected papers, World Scientific (2001)

[HiKuPoStr93] Hida T., Kuo H.-H., Potthoff J., Streit L.: White Noise. An Infinite Dimensional Calculus, Kluwer Academic Publishers (1993) 185231

[Hi75] Hida T.: Analysis of Brownian Functionals, Carleton Mathematical Lecture notes 13 (1975); 2nd ed., 1978

[HuPa84b] Hudson R.L., Parthasarathy K.R.: Construction of quantum diffusions, in: Quantum Probability and applications to the quantum theory of irreversible processes, L. Accardi, A. Frigerio, V. Gorini (eds.) Springer LNM N 1055 (1984)

[KonLit00] Y. Kondratiev, E. Lytvynov Operators of gamma white noise calculus Infin. Dimen. Anal. Quant. Prob. Rel. Top. 3 303-335 2000

[KoSiStr97] Y. Kondratiev, J.L. Silva, L. Streit Generalized Appell Systems, Methods Funct. Anal. Topology 3 (3) (1997) 28-61

[LYTV02a] Eugene Lytvynov: Polynomials of Meixner's type in infinite dimensions Jacobi fields and orthogonality measures, arXiv:math.CA/0203026 v5, 27 May (2002).

[LYTV02b] Eugene Lytvynov: Orthogonal decompositions for Levy processes with an application to the gamma, Pascal and Meixner processes, arXiv:math.PR/0204087 v5, 27 May (2002).

[LYTV95c] E. Lytvynov: Multiple Wiener integrals and non-Gaussian white noises: a Jacobi field approach, Methods Func. Anal. and Topol. 1 (1995) 61-85.

[Meix34] Meixner J.: Orthogonale Polynomsysteme mit einen besonderen Gestalt der erzeugenden Funktion, J.Lond. Math. Soc. 9 (1934) 6-13

[NUSCH00] D. Nualart, W. Schoutens: Chaotic and predictablerepresentations for Lévy processes, Stochastic Process. Appl. 90 (2000) 109-122

[Parth92] Parthasarathy K.R.: A quantum stochastic approach to Ito's formula for Lévy processes, C.R. Acad. Sci. Paris 315 Séries I (1992) 14171420

[Schou00] W. Schoutens: Stochastic Processes and Orthogonal Polynomials, Lecture Notes in Statist., Vol. 146, Springer-Verlag, New York (2000). 
[SchTeu98] W. Schoutens, J.L. Teugels: Lévy processes, polynomials and martingales, Commun. Statististics-Stochastic Models 14 (1998) 335-349

[Schü93] Schürmann, M.: White noise on bialgebras. Springer Lect Notes Math: 1544 (1993)

[Sega70a] Segal I.E.: Transformations in Wiener space and squares of quantum fields, Adv. in Math. 4 (1970) 91-108

[Śnia99] P. Śniady: Quadratic bosonic and free white noises, Commun. Math. Phys. 211 (3) (2000) 615-628 Preprint (1999)

[TsiVeYo01] N. Tsilevich, A. Vershik, M. Yor: An infinite-dimensional analogue of the Lebesgue measure and distinguished properties of the gamma process, J. Funct. Anal. 185 (2001) 274-296 